\title{
Modeling Biological Systems Using Functional State Spaces
}

\author{
Andy E. Williams, Nobeah Foundation, Nairobi, Kenya
}

\begin{abstract}
:
The technique of Human-Centric Functional Modeling represents systems as having a set of functions through which the system might transition from one state to another. These are functional states in that each state is described in terms of the functions available to transition to adjacent states. All possible behaviors of the system are then described by a graph containing a network of nodes representing such functional states, where those nodes are connected by edges representing the processes through which those functional states transition between each other. This paper outlines a hypothesis regarding how biological systems might come to occupy such a graph, also called a functional state space, and outlines some questions that must be answered in order to validate this hypothesis.
\end{abstract}

\section{Introduction}

This paper leverages the concept of "functional state spaces" which the author originated independently, though there is a tradition in philosophy that has already pioneered a similar concept. Bas C. van Fraassen, the philosopher to whom others [1] have attributed credit for the concept of the "state space" [2] as a semantic modeling approach, continues to this day to pioneer work on this and related topics. Van Fraassen and number of researchers citing his work have applied that concept of state spaces to biology [1], physics [3], and other subjects as this author has attempted to do. The difference appears to be that this author has made the additional refinement of restricting each state space to a single domain of behavior (to a single domain of functionality), which requires defining states solely in terms of the functionality each system is capable of from those states (that is, it requires defining states as "functional states"). The author has also used the human system, particularly the cognitive system, as a basis for this state space modeling approach, so that these models could be understood intuitively by first person introspective observation (by looking "inwards") without the need to understand any logical frameworks at all. Hence the name "Human-Centric Functional Modeling" or HCFM. This evolution from Bas C. van Fraassen's concept of "state space" to the concept of an intuitive "functional state space" has facilitated much clearer insight about how properties of each system in each domain of behavior might be defined in terms of these functional state spaces. Since these properties include complexity of the system and general problem-solving ability of the system (ability to solve any problem of transitioning from one functional state to another), since functional state spaces apply to systems relevant to a wide range of disciplines as described above, and since functional state is predicted to enable us to exponentially increase our ability to understand and navigate complexity as well as to solve problems in these systems where not otherwise reliably achievable, and since it is predicted that importance of a discovery can be measured in terms of the volume of functional state space the discovery enables to be navigated, this discovery of functional state spaces is predicted to be the most important innovation in the world today in a wide range of scientific disciplines. However, Bas C. van Fraassen's contribution remains invaluable. HCFM has been used to develop a model of group cognition called "General Collective Intelligence" or GCI [8] that is predicted to facilitate this exponential increase in our collective general problem-solving ability (intelligence) that is, to exponentially increase our ability to solve any problem in general to the point that many "wicked problems" in biology as well as problems in other areas that were previously unsolvable might be reliably solved [?]. GCI requires the world be described in terms of these "functional state spaces" which are based on van Fraassen's ideas.

GCI can potentially enable researchers to self-assemble into massive networks of cooperation with the capacity to radically increase impact in understanding biological systems, and to do so in a self- 
sustaining way. Because of this, the only bottleneck to massively increasing implementation of GCI is not predicted to be funding but instead is predicted to be mind share. An analogy is that there are crewless ships waiting at the dock full of provisions and ready to take us on towards our destination half way around the world. We just have to train a crew to sail them. In the same way, GCI might be an engine for global change, and when enough mind share is created, that engine will self-ignite. By virtue of the fact that van Fraassen might be the most highly cited author in the world on that topic, he has likely been more important in building mind share for the concept of state spaces than anyone else.

Here we hypothesize how such functional state spaces might arise. Assume that some number $\mathrm{N}_{1}$ of biological structures (for example cells or sub-cellular structures) come together through evolution or some other adaptive process to form some function $\mathrm{F}_{1}$ with the capability to achieve some outcome. Assume that the number of functions possessed by the set of structures grows after additional evolution when some number $\mathrm{N}_{2}$ of the same or different biological structures come to form some additional function $\mathrm{F}_{2}$ with the capability to achieve some state that is somehow related in that it in the same category or "space" in terms of impacting the same outcome as that achieved by $F_{1}$. As the set of functions increases, at some point the number of functions with which the biological organism can navigate this "space" might come to "span" that entire possible space, at which point the organism can potentially navigate the entire space of possible states through that set of functions. Assume the goal of living organisms is to change their internal state in order to maintain stability in their fitness (wellbeing) in the external environment, whether this means changing internal state in a way that generates some form of locomotion in order to move to a different region in the environment that has more resources or fewer waste products, or whether it means changing internal state in a way that varies the internal chemistry of the organism so that substances from the environment are caused to be either absorbed or excreted. If so, then this sudden expansion of the functional state space that can be navigated by the organism that occurs when the set of biological functions grows to "span" an entire "functional space" is an occurrence that might be detected as a sudden increase in fitness by the evolutionary or other adaptive process governing that set of structures defining this "system" within the organism. As an example, assume the need for water was a common element in a large number of biological processes that an organism might acquire to increase its fitness within some system, and assume the organism first evolved a function $F_{1}$ that enabled it to find water on top of the ground, then evolved a function that enabled it to find water underground, and so forth until it came to have set of functions $\left[\mathrm{F}_{1}, \mathrm{~F}_{2}, \mathrm{~F}_{3}, \mathrm{~F}_{4}\right]$ within this system that enabled it to find water everywhere. This increase in the functional state space that arises from the set of functions coming to span the entire functional state space would be detected by the evolutionary process as a sudden increase in the availability of water to that system. This increase in availability of a resource upon which many potential processes depend would enable many more evolutionary branches of that system to be explored.

In order for this navigation through functional state space to be stable, the system must navigate that functional state space in a way that ensures its continued fitness to execute all of its functions. Assume that if a set of functions "spans" a given domain represented by a specific functional state space, then each activity in that domain can be composed from some sequence of that basic set of functions. The sequence of activities selected to be executed by the system must function to maintain stability in terms of both tending to keep the fitness of the system within a stable range, and not allowing the system to exceed the range past which stability can no longer be recovered. By executing activities in a sequence that keeps the fitness (well-being) of the system within a stable range, the process representing that system then navigates the functional state space of the system as well as potentially navigating the external environment. 
All biological systems can potentially be seen as adaptive problem-solving processes that operate in some domain represented by this functional state space. A problem in functional state space is defined as the lack of a path from one functional state to another. A solution in functional state space is defined as the set of activities which provides that path. Considering each system to be an adaptive system, if that system has the potential to "adapt" in terms of coming to solve any problem in general that might be posed by the environment and doing so through acting within its domain as a system, systems can be seen to have general problem-solving ability within their domain. In order to have general problemsolving in their domain, each system must have the potential ability to navigate its entire functional state space while maintaining stability of its fitness to continue to execute its functions. Since each process executed by the system consumes resources, the need to maintain this dynamic stability must guide the selection of which subsequent activity the system will execute. This suggests some "convection" between investing more resources in the current activity, or searching for a new one.

\section{Convection Between Current Process Step and Incentive and Next Process Step}

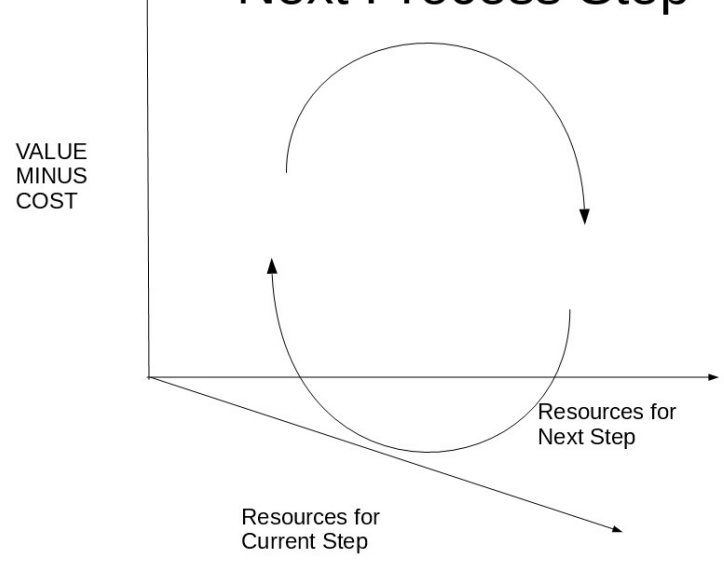

Defining a "fitness space" in terms of the actual fitness of the activity in achieving the targeted outcome, the predicted fitness of the activity to achieve the targeted outcome, and the targeted fitness of the activity to achieve the targeted outcome, this convection would be expected to arise explicitly to keep the behavior within a bounded region of fitness. One set of equations that display dynamical stability in terms of remaining within a bounded region in three dimensions are the Lorenz equations for convection, since the parameters of the Lorenz equations can be chosen to form globally stable dynamics (a strange attractor) in a three dimensional space. Designing a system so that it is governed by such dynamics would provide dynamical stability in the fitness space of the system, despite the path through the functional state space of the system potentially being chaotic. By defining each process in terms of a set of basic functions to create a library of such processes, and by defining a model for the fitness of each process, biological systems might then be modeled by an algorithm defined to select these activities in a way that maintains this dynamical stability where that stability is defined according to some set of equations exhibiting such stability.

In this way, from the perspective of Human-Centric Functional Modeling, the term "system" in biology is defined by some set of components which together executes some set of functionality that together comprise the complete set of behaviors possible for that system, and which executes that functionality in a dynamically stable way. The system then navigates that functional state space. This functional state space containing the complete set of behaviors of that system might be open in the sense that new states can be added or existing states removed, or it might be closed. 


\section{Mapping the Functional State Spaces of Organisms}

It has been suggested that every "system" within any biological organism can be represented by a functional state space. However, of all possible systems that organisms might be seen as being comprised of, only in the case of the cognitive system can the behaviors of that system be assured to be conceptualizable by definition since the functional state space occupied by the cognitive system is a space of concepts or a "conceptual space", since every behavior in the functional state space of the cognition is a reasoning process, and since every reasoning process is represented by a concept. Some of the other systems thought to exist in biological organisms and the capacities they provide are listed in table 1 .

\begin{tabular}{|l|} 
System (Capacity Provided by System) \\
\hline Homeostasis (Capacity to Achieve Stability) \\
\hline Autopoiesis (Capacity to Reproduce) \\
\hline Growth (Capacity to Maximize Outcomes) \\
\hline $\begin{array}{l}\text { Store Configuration or Genetic (Capacity to Store and Evolve } \\
\text { Configuration) }\end{array}$ \\
\hline $\begin{array}{l}\text { Sensory-Motor System (Capacity to Act) and Consciousness System } \\
\text { (Capacity for Choice) }\end{array}$ \\
\hline $\begin{array}{l}\text { Cooperation System (Capacity for Multicellular Cooperation or Cooperation } \\
\text { Between Functional Units) }\end{array}$ \\
\hline Behavior Storage System (Capacity to Learn Behaviors) \\
\hline Emotional System (Capacity to Prioritize Behaviors) \\
\hline Cognition (Capacity to Reason) \\
\hline
\end{tabular}

Table 1: A proposed hierarchy of systems in organisms.

The phenomenon of "choice" is represented as developing functionality that enables the system to switch between multiple patterns of stable dynamics in fitness space. Figure 1 depicts dynamics in fitness space of a functional component with three stable paths in fitness space and with a "choice" process $F_{S}$ to select between them. Each of these stable paths $\left[F_{1}, F_{2}, F_{3}\right]$ might correspond to a different activity in the same functional state space, or it might correspond to a different activity in an entirely different functional state space. The detection of position in functional state space and this choice of activity to execute enables the system to navigate a single larger functional state space or to navigate multiple functional state spaces. When an organism has developed the capacity to "choose" rather than to simply respond it is defined in this Human-Centric Functional Modeling approach as having developed the ability to be aware, that is, it has developed a rudimentary consciousness. When it has consciousness, it is defined in this Human-Centric Functional Modeling approach as having developed the ability to "act". 


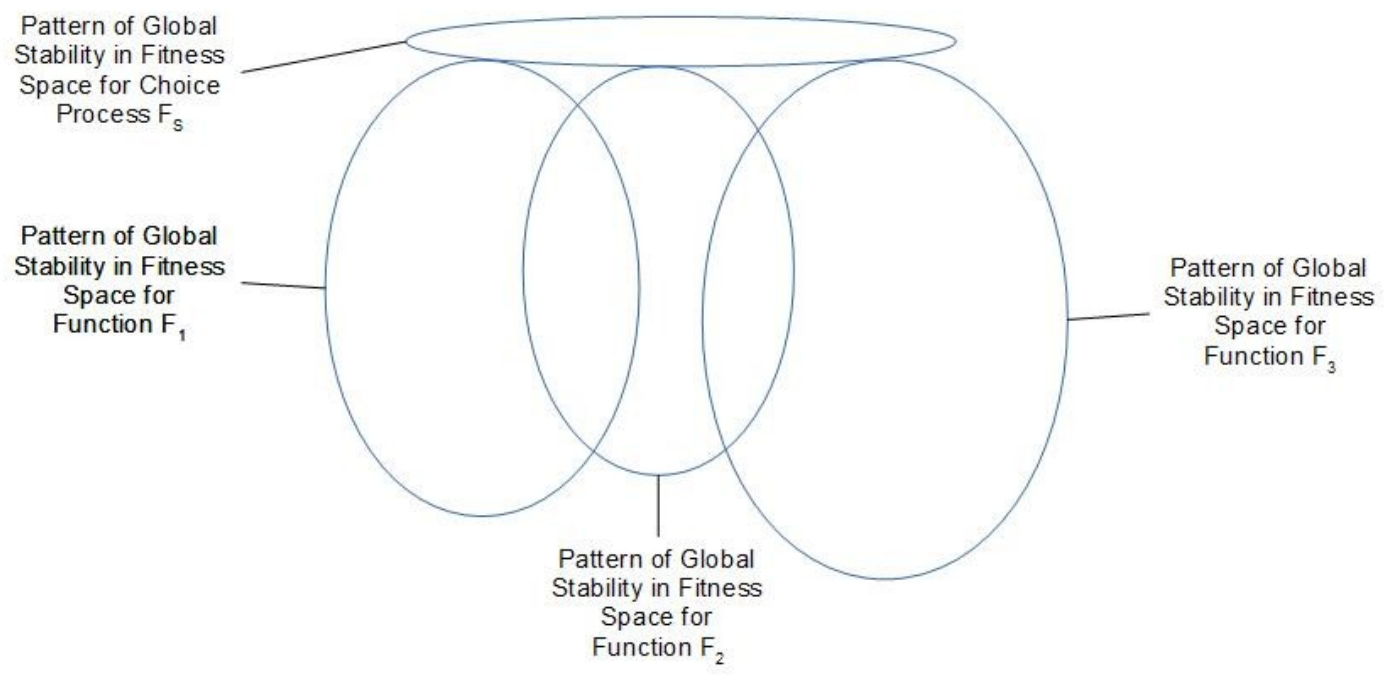

The existence of these systems and their order in this hierarchy was deduced from a number of assumptions and anecdotal observations. One assumption was that some set of functions must be associated with the stable ability of an organism to achieve each new category of outcome, and that the ability to execute each set of functions with general problem-solving ability in that problem domain is the definition of a system. Another assumption was that any system required by another system must have evolved earlier than that second system. The anecdotal observations were that from an informal survey of existing organisms, some organisms were observed to have lower systems without having higher systems, but none were observed to have higher systems without having lower systems, which appeared to confirm this hierarchy. As an example, some organisms can be habituated to exhibit behaviors, which is a simple form of learning [4], but lack a nervous system to receive sensory inputs and therefore could not be assumed to have the capacity to reason if reasoning is defined as having the capacity to "conceptualize" sensory inputs. A formal study confirming this hierarchy remains to be performed.

The systems in this hierarchy that operate "consciously" are hypothesized to be broken down further as in table 2.

\begin{tabular}{|ll|}
\hline System & Sub-System \\
\hline Sensory-Motor System & Tactile System \\
& Auditory System \\
\cline { 2 - 2 } & Gustatory System \\
\cline { 2 - 2 } & Olfactory System \\
\hline Eisual System \\
\hline Cognitive System & \\
\hline Consciousness System & \\
\hline
\end{tabular}

Table 2: A proposed hierarchy of conscious systems in the human organism. 


\section{Communication Between Systems}

Once one system has through evolution solved the problem of discovering how to interact with another system within the organism, the functions used to achieve that interaction might potentially be reused by any later system that evolves from it. Whether those functions might also come to be incorporated into systems that evolved earlier remains to be explored. As an example, since humans can be observed to have the capacity to be conscious of any sensory state, and assuming that all sensory states can be represented as some point within a "sensory-motor state space" representing the functional state space of the sensory-motor system, then the consciousness system can potentially be represented as having the ability to access every point in that sensory-motor state space. Each sensory-motor state represents a sensation. Assuming that only the sensory-motor system can move through sensory-motor states (i.e. only the sensory-motor system can take on sensory-motor states) then rather than interacting through taking on those states, other system will have to interact with sensations as external signals. There are potentially only three functions required to detect any signal in a field of signals generated by an array of sensory receptors: one function to determine the position of the signal in the array of sensory receptors, one function to determine the sequence of signals, and one function to determine the pattern in the sequence. Once these functions have been discovered by a primitive consciousness system for the purposes of interacting with tactile sensations for example, they might be available for reuse in interacting with visual, auditory, and other sensory input. They also then might be available for reuse in evolving the emotional system, as well as in evolving the cognitive system.

\section{Discussion}

The potential usefulness of defining functional state spaces for biological processes is that every path describes a model for a biological function that is universal in that it depends only on observed functionality and not on any assumption of how that functionality might be implemented. Once every process can be modeled as a path between one point in that space and another, then every proposed mechanism describing how that functional model might be implemented can potentially be compared in terms of its fitness to achieve the targeted outcome where incompatibilities between models might remove the possibility of such comparisons reliably being made today. Such universal comparability potentially enables all biological research to converge on a single understanding at radically greater speed and scale.

Functional state space is essentially the representation of a system as a network. As network science is today established as a backbone for description of structure and function of various physical, chemical, biological, technological, and social systems [5], functional state spaces can potentially be used to represent a wide variety of systems. Accordingly, this same pattern of defining a functional state space for a system and using that functional state space for increasing our collective capacity to understand and navigate the complexity of that system has been explored for multiple types of systems in a variety of other disciplines outside biology. In the case of the individual cognitive system this pattern has been proposed for use in defining functional models of the intuitive or methodical reasoning processes that might be used to represent all current AI solutions, and in combining all implementations of those solutions into a library that a model of artificial cognition [6] might use to continually increase its general problem-solving ability [7]. This pattern has also been proposed for use in combining all implementations of collective reasoning processes into a library that a collective reasoning platform such as a "General Collective Intelligence" [8] might use to continually increase its general problemsolving ability. In addition to exploring how this pattern might be implemented in cognitive systems for which the functions can by definition be conceptualized, this pattern is also being explored with other systems for which the functions must be deduced in other ways. One example is exploring how the entire physical universe or any object in it might be represented in terms of such functional state spaces in order to radically increase our ability to understand the physics of it. The basic functions in the case 
of this physical matter in our observable universe are hypothesized to be the four fundamental forces, which cannot be determined through observation of our own cognitive processes and instead must be determined through experimentation. The functions in the parts of our universe that are not observable and that might be represented by other functional state spaces are functions that might be determined through other kinds of experimentation such as searching for invariant properties in other functional state spaces that might conceivably result in forces within those spaces. Another example is applying functional state spaces to software in order to radically increase our ability to achieve some outcome [9], such as simultaneously achieving massive increases in the decentralization, security, and scalability of blockchain platforms.

In any case, representing biological systems in terms of functional state spaces appears to have the potential to represent complex biological systems in different resolutions in both time and length scales, which has been found to be very useful in modeling the action of complex systems [10].

The hierarchy of functional models defined through this Human-Centric Functional Modeling might define a hybrid modeling frameworks in that it is hypothesized to be capable of combining deterministic and stochastic, discrete and continuous, as well as multi-timescale features. Others have expressed the desirability of establishing hybrid modeling frameworks for these purposes [11]. Such hybrid frameworks might contain a range of representations, such as Structure-Behavior-Function (SBF) representations, which one study suggests may enable more accurate inferences about biological systems for complex and abstract questions than purely textual, or textual and diagrammatic, representations, and that no one representation is best for all types of inferences [12]. Other efforts have also attempted to achieve the reconciliation of different model types into a single composite representation by formulating a unifying theme that transcends traditional boundaries between disciplines [13]. It remains to be determined which lessons from those efforts might be reused in defining biological systems in terms of this hierarchy of functional state spaces.

Functional models are also dynamical models in that they represent the dynamics of the system that result from executing its functions. Dynamical modeling is important in overcoming the barriers to understanding biological complexity that occur when systems have too many elements, too many interactions between those elements, and when the resolution at which those interactions must be understood is too great to fit within the space of human cognition [14]. Some questions that remain as this approach of Human-Centric Functional Modeling is used to elaborate functional models occupying such functional state spaces is what is the relationship between functional state spaces and other spaces such as "phase space" that have been used to represent biological systems [15]?

Discovering the basic set of functions in each hypothesized functional state space remains an unexplored challenge. The functions in cognition might be discovered through introspection of our own cognition as has been proposed in one model [6], but what are the functions that might make up the hypothetical homeostasis system for example? Or the genetic system? The recent advent of so-called genome-wide high throughput methods have generated the called omics data (e.g. metabolomics, proteomics, transcriptomics and genomics) of bio-molecules like mRNA, proteins and metabolites, the detection of molecular interactions, such as, protein-DNA and protein-metabolite have allowed a better understanding of the regulation of metabolism at a global scale and might permit this kind of wholecell computational model that integrates different omics data, and that integrates the different approaches for the model-driven interpretation of these data [16], [17].

\section{Conclusion}


It remains to be confirmed that each biological system can be represented in terms of a set of basic functions that span some functional state space describing the entire set of behaviors of that system. It also remains to determine what these functions are and to demonstrate that these functions can be used to define functional models that essentially serve as unique identifiers (primary keys) in a database of all biological processes that facilitates convergence in our understanding of those processes. However, the motivations for answering these questions are potentially great. Whether all thought about biological systems is represented in a "conceptual space" (the functional state space of the cognitive system) that provides a semantic representation of all information which enables an artificial cognition or a General Collective Intelligence to radically increase our ability to solve problems in biology, or whether the biological systems themselves are represented in terms of functional state spaces that radically increase our capacity to navigate biological processes, in either case the use of functional state spaces, if it applies, is hypothesized to have the capacity for great impact on the advance of biological science.

\section{References}

[1] Lloyd, Elisabeth A. "A semantic approach to the structure of population genetics." Philosophy of Science 51.2 (1984): 242-264.

[2] Van Fraassen, Bas C. "On the extension of Beth's semantics of physical theories." Philosophy of science 37.3 (1970): 325-339.

[3] Van, Fraassen, and Bas C. Van Fraassen. Quantum mechanics: An empiricist view. Oxford University Press, 1991.

[4] Romain P. Boisseau, David Vogel, Audrey Dussutour. Habituation in non-neural organisms: evidence from slime moulds. Proceedings of the Royal Society B: Biological Sciences, 2016; 283 (1829): 20160446 DOI: 10.1098/rspb.2016.0446

[5] Gosak M, Markovič R, Dolenšek J, Slak Rupnik M, Marhl M, Stožer A, Perc M. Network science of biological systems at different scales: A review. Phys Life Rev. 2018 Mar;24:118-135. doi: 10.1016/j.plrev.2017.11.003. Epub 2017 Nov 3. PMID: 29150402.

[6] Williams A.E. (2020) A Model for Artificial General Intelligence. In: Goertzel B., Panov A., Potapov A., Yampolskiy R. (eds) Artificial General Intelligence. AGI 2020. Lecture Notes in Computer Science, vol 12177. Springer, Cham. https://doi.org/10.1007/978-3-030-52152-3_38

[7] Williams, A. E. (2020, April 27). Defining Functional Models of Artificial Intelligence Solutions to Create a Library that an Artificial General Intelligence can use to Increase General Problem Solving Ability. https://doi.org/10.31730/osf.io/hpzb7

[8] Williams, A. E., Defining a Continuum from Individual, to Swarm, to Collective Intelligence, to General Collective Intelligence, International Journal of Collaborative Intelligence, in press (2021)

[9] Andy E. Williams, "Cognitive Computing and its Relationship to Computing Methods and Advanced Computing from a Human-Centric Functional Modeling Perspective", In: Pal R. and Shukla K. P. (eds), SCRS Conference Proceedings on Intelligent Systems, SCRS, New Delhi, India, 2021, pp. 16-33. https://doi.org/10.52458/978-93-91842-08-6-9 
[10] Warshel, A. (2014), Multiscale Modeling of Biological Functions: From Enzymes to Molecular Machines (Nobel Lecture). Angew. Chem. Int. Ed., 53: 10020-10031.

https://doi.org/10.1002/anie.201403689

[11] Wu, J., Voit, E. Integrative biological systems modeling: challenges and opportunities. Front. Comput. Sci. China 3, 92-100 (2009). https://doi.org/10.1007/s11704-007-0011-9

[12] Helms, M, Vattam, S, \& Goel, A. "The Effect of Functional Modeling on Understanding Complex Biological Systems." Proceedings of the ASME 2010 International Design Engineering Technical Conferences and Computers and Information in Engineering Conference. Volume 5: 22nd International Conference on Design Theory and Methodology; Special Conference on Mechanical Vibration and Noise. Montreal, Quebec, Canada. August 15-18, 2010. pp. 107-115. ASME. https://doi.org/10.1115/DETC2010-28939

[13] Broderick G, Rubin E: The Realistic Modeling of Biological Systems: A Workshop Synopsis. ComPlexUs 2006;3:217-230. doi: 10.1159/000106145

[14] Ruth M., Hannon B. (1997) Modeling Dynamic Biological Systems. In: Modeling Dynamic Biological Systems. Modeling Dynamic Systems. Springer, New York, NY. https://doi.org/10.1007/978-1-4612-0651-4_1

[15] R.J. Bagley, J.D. Farmer, S.A. Kauffman, N.H. Packard, A.S. Perelson, I.M. Stadnyk, Modeling adaptive biological systems, Biosystems, Volume 23, Issues 2-3, 1989, Pages 113-137, ISSN 03032647, https://doi.org/10.1016/0303-2647(89)90016-6.

[16] Costa, R. S., Machado, D., Neves, A. R., \& Vinga, S. (2012). Multi-level dynamic modeling in biological systems: Application of hybrid Petri nets to network simulation. In Proceedings of the International Conference on Bioinformatics Models, Methods and Algorithms (pp. 317-321). SciTePress. https://doi.org/10.5220/0003785503170321

[17] Lore Cloots, Kathleen Marchal, Network-based functional modeling of genomics, transcriptomics and metabolism in bacteria, Current Opinion in Microbiology, Volume 14, Issue 5, 2011, Pages 599607, ISSN 1369-5274, https://doi.org/10.1016/j.mib.2011.09.003.

[18] Williams, A.E. Are wicked problems a lack of general collective intelligence?. AI \& Soc (2021). https://doi.org/10.1007/s00146-021-01297-8 